\title{
Treatment outcomes of pulmonary multidrug-resistant tuberculosis: 10 year follow up study
}

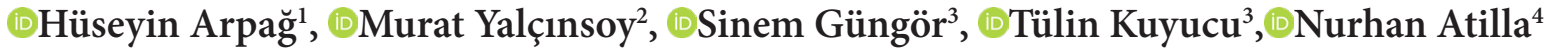 \\ ${ }^{1}$ Batman Medical Park Hospital, Department of Chest Diseases, Batman, Turkey \\ ${ }^{2}$ Inonu University Turgut Ozal Medical Center Malatya, Turkey \\ ${ }^{3}$ Süreyyapaşa Chest Diseases and Chest Surgery Training and Research Hospital, Chest Diseases Clinic, İstanbul, Turkey \\ ${ }^{4}$ Kahramanmaras Sutcu Imam University Medicine Faculty, Department of Chest Diseases, Kahramanmaraş, Turkey
}

Cite this article as: Arpağ H, Yalçınsoy M, Güngör S, Kuyucu T, Atilla N. Treatment outcomes of pulmonary multidrug-resistant tuberculosis: 10 year follow up study. Anatolian Curr Med J 2021; 3(1); 26-30.

\begin{abstract}
Aim: Treatment rates of multidrug-resistant tuberculosis (MDR-TB) vary from clinic to clinic. In this study, the characteristics of MDR-TB patients followed in two clinics of our hospital and their relationship with treatment results were investigated.

Material and Method: This observational retrospective cohort study performed in a government hospital which is one of the important reference center for management of tuberculosis in Turkey. Seventy-nine adult MDR-TB cases who'm diagnosed and treated between 2004-2014 were retrospectively enrolled into the study.

Results: Of the patients with MDR-TB, 58 were male, 21 were female, mean age was $36.24 \pm 13.75$ and all of them were HIV (-). 64 patients completed treatment, 15 patients still under treatment. The average hospitalization time was 162.76 . Radiologically, 11 patients had diffuse disease and 68 patients had limited disease. The mean number of patients who received second-line drugs were $5.18 \pm 0.5$ and drug-related side effects developed in 36 patients. Cure was achieved in $84.4 \%$ of the patients, $7.8 \%$ discontinued the treatment, $7.8 \%$ died during the treatment and there wasn't any treatment failure.

Conclusion: In our study, we think that the factors that increase the success of MDR-TB treatment are: young age, low resistance to second line medication, prolongation of hospitalization, good compliance with treatment, low rate of ofloxacin use in previous treatment, use of fluoroquinolones in new patients, surgery in selected patients.
\end{abstract}

Keywords: Multidrug-resistant, pulmonary, tuberculosis

\section{INTRODUCTION}

Multidrug-resistant tuberculosis (MDR-TB) is a global serious disease. The term of MDR-TB refers to an isolate of Mycobacterium Tuberculosis (M.TB) that is resistant to at least isoniazid $(\mathrm{H})$ and rifampicin $(\mathrm{R})$ and possibly additional agents (1). It has many different properties than non-resistant and single drug resistant tuberculosis (TB) forms. Mortality from MDR-TB is high, and second/third-line therapeutic agents are less potent, less tolerable, less readily available, and more costly than first-line therapies. The MDR-TB patients require longer periods of treatment than other TB forms. Due to these reasons, it is very important to investigate and determine characteristics and outcome of MDR-TB. However data on MDR-TB from developing countries are limited (2).

MDR-TB is a difficult detectable mortal disease. According to 2015 World Health Organization (WHO) TB report; of the 480.000 cases of MDR-TB estimated to have occurred in 2014, but only about a quarter of these patients (123.000) were detected and reported. So, there are gaps in the disease reporting. An estimated 3.3\% of new TB cases and $20 \%$ of previously treated cases have MDR-TB. It is estimated that 190.000 people died from MDR-TB in 2014.

World Health Organization categorized the countries as five regions in global TB report. Turkey is located in European (EUR) region with similar incidence of Europian countries. There are limited studies about disease charecteristics, outcomes and long term treatment results of MDR-TB in Turkey (2). In 2015 the number of MDR-TB cases reported from Turkey was 359 (2). In the present study, we planned to evaluate the characteristics and treatment outcomes of MDR-TB cases in our hospital which is an important reference center for management of tuberculosis patients. 


\section{MATERIAL AND METHOD}

\section{Patients}

This observational retrospective cohort study was performed in a spesific thoracic diseases hospital which is one of the important reference center for management of tuberculosis in İstanbul, Turkey.

Sixtyseven MDR-TB patients who treated with standard protocol (PAS, Cycloserin, Prothionamid, Amikacin, Quinolon), and 12 treated with special protocol (Moxifloxacin, Thiosetazon, Pyrazinamide, Amoxicillin/ Clavulanic acid, Cycloserine, Clofazimine, Capreomisin, Linezolid) between the 2004-2014 were retrospectively enrolled into the study. The study was approved by local Ethics Committee of the Institution (2014/1). The study was conducted in accordance with the principles of Declaration of Helsinki.

Data of demographic profile, medical history, comorbidities, smoking habits, purified protein derivative (PPD) measurements, index case features, clinicalradiological findings, sputum smear examination, drug susceptibility pattern, previous treatment details, drug side effects, duration of hospitalization, surgical requirements and cause of death were noted from hospital archive records.

Sputum microscopy evaluated with Ziehl-Neelsen technique, and sputum cultures evaluated by Lowenstein-Jensen medium. MDR-TB was diagnosed when M.TB isolates were resistant to both $\mathrm{H}$ and R. Drug susceptibility test (DST) was performed on LowensteinJensen medium by proportion method and liquid broth automated systems.

\section{Treatment Regimen}

Patients were treated in accordance with the general MDR-TB treatment regimens. After the diagnosis, at least five MDR-TB drugs (para-aminosalicylic acid, capreomycin, puromycin, cycloserine, ofloxacin, moxifloksasin, amikacin, clofazimine, linezolid, prothionamide, thioacetazone, amoxicillin-clavulanate and clarithromycin) were included in the treatment regimen.

Patients were hospitalized until sputum acid fast bacilli (AFB) smears were negative for three consecutive days. After discharging, drugs were given regularly by directly observed treatment (DOT) programme. Follow up evaluations included complete blood counts, renal and liver function tests, chest $\mathrm{X}$ ray, sputum smear and culture examinations monthly. Achievement of sputum AFB negativity in the 3 rd month of the treatment was accepted as the bacteriologic response. Treatment was continued for at least 18 months after the first negative culture obtained.
Definitions related drug resistance, radiological findings and treatment results

Primary resistance: Drug resistance in cases previously not used TB drugs or used shorter than one month.

Secondary resistance: Drug resistance in patients who previously used TB drugs at least one month.

Index: A contagious case who is source for TB or MDRTB infection.

Limited disease: Infiltrates involving less than $25 \%$ of the lung fields or the presence of cavities totaling less than 15 $\mathrm{cm}$ in diameter (3).

Extensive disease: Infiltrates involving at least $75 \%$ of the lung fields or the presence of cavities totally at least $15 \mathrm{~cm}$ in diameter (3).

Cured: Patient who completed the course of antituberculosis treatment (ATT) and was culturenegative in the last month of treatment and had been culture-negative during the preceding 11 months of treatment.

Treatment completed: A patient who completed treatment but did not meet the definition for cure or failure due to lack of bacteriologic results.

Treatment default: A patient whose treatment was interrupted for two or more consecutive months due to any reason, including patients who left against medical advice.

Treatment Failure: A patient who had more than one positive culture in the last 12 months of treatment, with a minimum of five cultures performed during the last 12 months, or a patient who remained persistently culturepositive and a clinical decision was made to terminate treatment early.

Death: A patient who died of any reason during treatment.

\section{RESULTS}

A total of 79 patients with mean age $36.24 \pm 13.75$ (18-70) years, included to the study male proportion was $73.4 \%$ (Table 1). Twenty seven (34.2\%) patients were TB index and 7 (9\%) patients were MDR index, 25 (31.6\%) patients had comorbidity, radiologic involvement was exstensive in $11(14 \%)$ and limited in $68(86 \%)$ cases and HIV serology was negative in all patients (Table 1). Thirty five (44.3\%) patients had primary, $44(55.7 \%)$ had secondary drug resistance (Table 2).

Sixty seven (84.8\%) cases underwent standard treatment protocol (PAS, Cycloserin, Prothionamid, Amikacin, Quinolon). Twelve (15.2\%) patients underwent special treatment (Moxifloxacin, Thiosetazon, Pyrazinamide, Amoxicillin/Clavulanic acid, Cycloserine, Clofazimine, Capreomisin, Linezolid) due to minor drug resistance or drug side effects. DST was obtained from all cases. Two patients underwent lobectomy. 


\begin{tabular}{|lc|}
\hline \multicolumn{2}{|l|}{ Table 1. Characteristics of MDR-TB patients } \\
\hline Characteristics & Patients \\
\hline Age (years), mean \pm SD & $36.24 \pm 13.75$ \\
Gender (F/M) & $2 / 58(26 \% / 74 \%)$ \\
Smoking history & $25(31.6 \%)$ \\
Aditional diseases & $25(31.6 \%)$ \\
HIV infected & None \\
Side effects & $36(45.5 \%)$ \\
Previously treated & $41(51.8 \%)$ \\
Previous treatment $>2$ years & $11(13.9 \%)$ \\
TB index case (+) & $27(34.2 \%)$ \\
MDR index case (+) & $7(9 \%)$ \\
Primary drug resistance & $35(44.3 \%)$ \\
Secondary drug resistance & $44(55.7 \%)$ \\
Extensive disease & $68(86 \%)$ \\
Cavity (+) & $64(81 \%)$ \\
Sputum conversion & $1.8 \pm 1.3$ \\
Culture conversion & $1.9 \pm 1.03$ \\
Surgery performed & $1(0.12 \%)$ \\
Mean hospital stay day & 162.76 \\
Treatment completed & 64 \\
Treatment ungoing & 15 \\
\hline SD: standard deviation, F: female, M: male, TB: tuberculosis, MDR: multi-drug resistant. \\
\end{tabular}

Table 2. Results of drug susceptibility testing of multidrug-resistant tuberculosis strains

\begin{tabular}{|lcc|}
\hline Medication & Susceptible (n, \%) & Resistant (n, \%) \\
\hline First-line drugs & & \\
HR & $57(72.2)$ & $22(27.8)$ \\
HRE & $59(71.7)$ & $20(28.3)$ \\
HRS & $71(89.9)$ & $8(10.1)$ \\
HRES & $50(63.3)$ & $29(36.7)$ \\
Second-line drugs ${ }^{*}$ & & \\
Ethionamide & $75(95)$ & $4(5)$ \\
Ofloxacin & $77(97.5)$ & $2(2.5)$ \\
Kanamycin & $78(98.8)$ & $1(1.2)$ \\
PAS & $77(97.5)$ & $2(2.5)$ \\
Rifabutin & $78(98.8)$ & $1(1.2)$ \\
\hline $\begin{array}{l}\text { *Minor drug resistance was seen in four patient. Multiple drug resistance has been } \\
\text { observed in several patients. } \\
\text { H: isoniazid, R: rifampicin, E: ethambutol, S: streptomycin, PAS: paraaminosalisilikacid }\end{array}$ \\
\hline
\end{tabular}

One or more drug-related side effects developed in 36 (45.5\%) patients; hearing loss (15), depression (12), psychosis (7) were most common side effects. Two cases attempted to suicide. Dermatitis, hepatotoxicity, nephrotoxicity and optic neuropathy were noted in a few cases (Table 3). Treatment discontinued due to drug toxicity in 11 cases against Amikacin, in 7 cases against cycloserine, in 1 case against linezolid. All drugs discontinued in 2 cases due to hepatotoxicity, treatment was restarted after the improvement of enzymes (Table 3).

\begin{tabular}{|lcc|}
\hline \multicolumn{2}{l}{ Table 3. Definition of drug dependent side effects } & \\
\hline Adverse effects & $\mathbf{n}$ & $\mathbf{\%}$ \\
\hline Hearing loss & 15 & 18.9 \\
Depression & 12 & 15.1 \\
Psychosis & 7 & 8.8 \\
Dermatitis & 4 & 5 \\
Hepatotoxicity & 3 & 3.7 \\
Nephrotoxicity & 1 & 1.2 \\
Optic neuropathy & 1 & 1.2 \\
\hline
\end{tabular}

Sixty four patients completed treatment, while 15 patients are still continuing to treatment. The mean duration time of the treatment was $24 \pm 4.2$ months. Mean hospital stay day was 162.76 days (30-436). Mean duration of sputum conversion was $1.8 \pm 1.3$ month, culture conversion was $1.9 \pm 1.03$ months. 64 patients completed the treatment. Cure was achieved in 54 patients (84.4\%), $5(7.8 \%)$ patients were treatment default, $5(\% 7.8)$ patients died (1 suicide, 1 hypoglycemic coma after hip fracture surgery, 1 bronchopleural fistula).

\section{DISCUSSION}

Multidrug-resistant tuberculosis is one of the most growing public health problem in all over the world. Treatment of the disease is very important and also difficult because of the risk of transmitting exactly untreatable drug resistant microorganism. There are so many factors that affecting treatment success such as; age, gender, comorbidities, patient compliance, side effects, treatment duration, degree of lung damage, high resistance to ATT, additional pulmonary complications, previous treatments etc. In our study, cure proportion was $84.4 \%$ of patients and this is a successful rate compared to other conducted locally and in regional countries studies (4-10). A comparison of our results with those of other studies conducted locally and in regional countries is given in Table 4.

\begin{tabular}{|lcccccc|}
\hline \multicolumn{2}{l}{ Table 4. Outcome comparison of multidrug-resistant tuberculosiscases in various studies } \\
\hline & Year & n & cure & death & default & failure \\
\hline Our study & $2001-2015$ & 64 & $54(84.4 \%)$ & $5(7.8 \%)$ & $5(7.8 \%)$ & 0 \\
Turkey**(4). & $1992-2004$ & 263 & $204(77.6 \%)$ & $18(6.8 \%)$ & $25(9.5 \%)$ & $16(6.1 \%)$ \\
Turkey ${ }^{*}(5)$. & $1998-2006$ & 64 & $34(53.1 \%)$ & $3(4.7 \%)$ & $18(28.1 \%)$ & $1(1.6 \%)$ \\
India(6). & $2006-2007$ & 38 & $25(65.8 \%)$ & $3(8 \%)$ & $5(13.1 \%)$ & $5(13.1 \%)$ \\
Pakistan(7). & $2007-2010$ & 40 & $3(10 \%)$ & $12(40 \%)$ & $9(30 \%)$ & $6(20 \%)$ \\
Taiwan(8). & $1992-1996$ & 299 & $153(51.2 \%)$ & $28(9.4 \%)$ & $87(29.1 \%)$ & $31(10.4 \%)$ \\
Russia(9). & $2000-2002$ & 244 & $186(77 \%)$ & $12(5 \%)$ & $29(12 \%)$ & $17(7 \%)$ \\
Bulgaria(10). & $2009-2010$ & 34 & $16(47.2 \%)$ & $13(38.2 \%)$ & $1(2.9 \%)$ & $4(11.7 \%)$ \\
\hline${ }^{*}$ Ankara, ${ }^{* *}$ Istanbul & & & & & \\
\hline
\end{tabular}


Previous studies found that being male was a risk factor for development of MDR-TB $(11,12)$. As compatible with the literature, most of our patients were male. Another study showed that males have an increased risk for MDR-TB, and being male was a risk factor for defaulting from ATT. Moreover, the same study showed that among MDR-TB cases who were defaulters in their first-line TB treatment, $62.5 \%$ were males (11). Likewise in our study all of treatment default patients were male. The association between being male and having MDR$\mathrm{TB}$ could be because males have a higher tendency to not adhere to ATT than females, thus increasing their risk of developing MDR-TB (13). Tahaoglu et al. (14) and Park et al. (15) reported that younger age is associated with successful outcome. In our study similarly to other studies big part of our patients were in productive age.

Treatment response rates in MDR-TB according to the HIV positivity were different in the previous studies. HIV positive patients were found to have lower response rate than HIV negative patients (16). Park et al. (17) evaluated 173 patients with MDR-TB, the majority of whom consisting of HIV positive patients. They found that mortality rates were significantly higher in HIV positive patients than HIV negative group. Unfortunately we couldn't compare the HIV positive and negative patients due to all of our patients were HIV negative.

The treatment of MDR-TB requires more complex regimen with longer treatment duration (18-24 months); thus, adverse drug events occur more frequently compared to the treatment regimen for nonMDR-TB (18). In some cases side effects can be serious enough to warrant discontinuation of the drug. Torun et al. evaluated 263 patients for the adverse effects of MDR-TB treatment retospectively. They found ototoxicity (41.8\%), psychiatric disorders (21.3\%), gastrointestinal disturbance $(14.0 \%)$ as the most frequent side effects. Similarly to Torun et al's study ototoxicity and psychiatric disorders were determined as the most frequent adverse effects in our patients (4).

Although treatment side effects are common in MDR$\mathrm{TB}$, effort should be made to continue treatment. In our study treatment changes were made in 20 patients because of serious side effects. Careful clinical monitoring, laboratory analysis and a multidisciplinary approach are essential in the management of side effects of MDR-TB cases.

Tahaoğlu et al. (14) reported that absence of ofloxacin in the previous treatments were associated with successful outcome. In our study only four patients were used ofloxacin in the previous treatments. Fluoroquinolones have considerable promise in the treatment of MDR-
$\mathrm{TB}$, especially the new generation fluoroquinolones (19). Fluoroquinolones are playing an important role in the treatment of MDR-TB. Thus, it is prudent to avoid indiscriminate use of fluoroquinolones in the treatment of respiratory infections before excluding TB because there is cross-resistance within the fluoroquinolone class (20), and incidental monotherapy of fluoroquinolone in TB can easily lead to fluoroquinolone resistance (21). All of our patients had new generation fluoroquinolone in the MDR-TB treatment and this may be an important reason for the high rate of bacteriologic response.

Two of our patients underwent right upper lobectomy due to persistent cavities in spite of ARB negativity. Cure was achieved in both of them. Resection surgery has been shown to be associated with a favourable outcome (22-24). Surgery should be considered as an adjunct to medical therapy when eradicating MDR-TB in affected patients. Early referral of such patients for surgical consideration is significant.

Mean hospitalisation duration of our patients were 162.7 days. We believe that long-term hospitalization increased the success of our treatment. In order to increase the success of treatment, MDR TB should be treated in specialized centers. Cure rate of our study was quite high and there wasn't any treatment failure. But 5 patients were treatment default. When we look at the other studies, we saw that default was seen more than treatment failure in most of the studies (5,7-9). Default from treatment, is the major trouble in the treatment of MDR-TB, that's why strategies to reduce number of defaulters are crucial in the treatment of MDR-TB.

Limitations: Since our study is single centered, it may not reflect the general public. Multicenter studies are needed on this subject.

\section{CONCLUSION}

The high cure rates of our study can be attributed to eight main factors 1) young population 2) HIV negativity in all patients 3) low resistance to secondline ATT medications 4) prolonged hospitalization 5) good patient adherence to treatment 6) low rates of ofloxacin use in previous treatment 7) the use of new fluoroquinolones in all patients 8) performing surgery in selected patients. We hope that knowing these factors that increasing the cure rates, will be beneficial for clinicians.

\section{ETHICAL DECLARATIONS}

Ethics Committee Approval: The study was carried out with the permission of local Ethics Committee (Permission granted 2014, Decision No. 2014/1). 
Informed Consent: Because the study was designed retrospectively, no written informed consent form was obtained from patients.

Referee Evaluation Process: Externally peer-reviewed.

Conflict of Interest Statement: The authors have no conflicts of interest to declare.

Financial Disclosure: The authors declared that this study has received no financial support.

Author Contributions: All of the authors declare that they have all participated in the design, execution, and analysis of the paper, and that they have approved the final version.

\section{REFERENCES}

1. Frieden TR, Fujiwara PI, Washko RM, Hamburg MA. Tuberculosis in New York City-turning the tide. N Engl J Med 1995; 333: 229.

2. World Health Organization. Global tuberculosis report, Geneva, Switzerland: WHO, 2015.

3. Centers for Disease Control and Prevention. Revised definition of extensively drug-resistant tuberculosis. MMWR Morb Mortal Wkly Rep 2006; 55: 1176.

4. Törün T, Güngör G, Özmen İ, et al. Side effects associated with the treatment of multidrug-resistant tuberculosis. Int J Tub Lung Dis 2005; 9: 1373-7.

5. Ünsal E, Güler M, Ofluoglu R, Capan N, Cimen F. Factors associated with treatment outcome in $64 \mathrm{HIV}$ negative patients with multidrug resistant tuberculosis. J Thorac Dis 2013; 5: 4359.

6. Nadu T, Pauline Joseph I, Bhaskara V, et al. Outcome of standardized treatment for patients with MDR-TB. Indian J Med Res 2011; 133: 529-34.

7. Khurram M, Tul H, Khaar B, Fahim M. Multidrug-resistant tuberculosis in Rawalpindi, Pakistan. J Infect Dev Ctries 2012; 6: 29-32.

8. Chiang CY, Enarson DA, Yu MC, et al. Outcome of pulmonary multidrug-resistant tuberculosis: a 6-yr follow-up study. Eur Respir J 2006; 28: 980-5.

9. Shin SS, Pasechnikov AD, Gelmanova IY, et al. Adverse reactions among patients being treated for MDR-TB in Tomsk, Russia. Int J Tuberc Lung Dis 2007; 11: 1314-20.

10. Milanov V, Falzon D, Zamfirova M, et al. Factors associated with treatment success and death in cases with multidrug-resistant tuberculosis in Bulgaria, 2009-2010. Int J Mycobacteriol 2015; 131-7.

11. Hirpa S, Medhin G, Girma B, et al. Determinants of multidrugresistant tuberculosis in patients who underwent first-line treatment in Addis Ababa: a case control study. BMC Public Health 2013; 13: 782.

12. Law WS, Yew WW, Chiu Leung C, et al. Risk factors for multidrug-resistant tuberculosis in Hong Kong. Int J Tuberc Lung Dis 2008; 12: 1065-70.

13. Heunis JC, Kigozi NG, van der Merwe S, Chikobvu P, Beyers N. Sex-related trends in non-conversion of new smear-positive tuberculosis patients in the Free State, South Africa. Public Health Action 2014; 4: 66-71.

14. Tahaoğlu K, Törün T, Sevim T, et al. The treatment of multidrugresistant tuberculosis in Turkey. N Engl J Med 2001; 345: 170-4.

15. Park MM, Davis AL, Schluger NW, et al. Outcome of MDRTB patients, 1983-1993. Prolonged survival with appropriate therapy. Am J Respir Crit Care Med 1996; 153: 317-9.
16. Goble M, Iseman MD, Madsen LA, Waite D, Ackerson L, Horsburgh CR Jr. Treatment of 171 patients with pulmonary tuberculosis resistant to Isoniazid and Rifampin. $\mathrm{N}$ Engl J Med 1993; 328: 527-32.

17. Park SK, Kim CT, Song SD. Outcome of chemotherapy in 107 patients with pulmonary tuberculosis resistant to isoniazid and rifampin. Int J Tuberc Lung Dis 1998; 2: 877-84.

18. Yew WW, Chan CK, Chau $\mathrm{CH}$, et al. Outcomes of patients with multidrug-resistant pulmonary tuberculosis treated with ofloxacin/levofloxacin-containing regimens. Chest 2000; 117: $744-51$.

19. Orenstein EW, Basu S, Shah NS, Andrews JR, Friedland GH, Moll AP. Treatment outcomes among patients with multidrugresistant tuberculosis: systematic review and meta-analysis. Lancet Infect Dis 2009; 9: 153-61.

20. Ginsburg AS, Grosset JH, Bishai WR. Fluoroquinolones, tuberculosis, and resistance. Lancet Infect Dis 2003; 3: 432-42.

21. Grimaldo ER, Tupasi TE, Rivera AB, et al. Increased resistance to ciprofloxacin and ofloxacin in multidrugresistant Mycobacterium tuberculosis isolates from patients seen at a tertiary hospital in the Philippines. Int J Tuberc Lung Dis 2001; 5: 546-50.

22. Chan ED, Laurel V, Strand MJ, et al. Treatment and outcome analysis of 205 patients with multidrug-resistant tuberculosis. Am J Respir Crit Care Med 2004; 169: 1103-9.

23. Chiang CY, Yu MC, Bai KJ, Lin TP, Lee YC. Pulmonary resection in the treatment of patients with pulmonary multidrug-resistant tuberculosis in Taiwan. Int J Tuberc Lung Dis 2001; 5: 272-7.

24. Park SK, Lee CM, Heu JP, Song SD. A retrospective study for the outcome of pulmonary resection in 49 patients with multidrugresistant tuberculosis. Int J Tuberc Lung Dis 2002; 6: 143-9. 